\title{
Bioassay
}

\section{Linha Básica de Suscetibilidade de Chrysodeixis includens (Walker, [1858]) (Lepidoptera: Noctuidae) a Benzoato de Emamectina}

\author{
Naiara R. Nunes ${ }^{1 \oplus}$, Fátima T. Rampelloti Ferreira ${ }^{1 \oplus}$, Leonardo V. Thiesen ${ }^{2 \oplus}$, Janaina $N$. \\ Corassa $^{1}{ }^{1}$, Rafael M. Pitta ${ }^{3= \pm=(0)}$
}

${ }^{1}$ Universidade Federal de Mato Grosso, Sinop-MT, Brasil. ${ }^{2}$ Faculdade de Ciências Agrárias e Veterinárias, Jaboticabal-SP, Brasil. ${ }^{3}$ Embrapa Agrossilvipastoril, Sinop-MT, Brasil.

莑=゙Corresponding author: rafael.pitta@embrapa.br

\section{Edited by: Leandro Delalibera Geremias}

Received: December 06, 2019. Accepted: December 12, 2019. Published: December 17, 2019.

Baseline susceptibility of Chrysodeixis includens (Walker, [1858]) (Lepidoptera: Noctuidae) to Emamectin Benzoate

Abstract. Emamectin Benzoate was registered recently in Brazil to control Chrysodeixis includens (Walker, [1858]) (Lepidoptera: Noctuidae) in soybean. We studied the baseline susceptibility of Mato Grosso populations of $C$. includes to support Insect Resistance Management programs by standard feeding assays on insecticide-surface of artificial diet. Variation in susceptibility between populations was low. The $\mathrm{LC}_{50}$ values from 11 populations varied between 0.015 a $0.065 \mu \mathrm{g}$ i.a. $\mathrm{cm}^{-2}$. Based on our results, we propose the discriminant concentration of $2.867 \mu \mathrm{g}$ i.a. $\mathrm{cm}^{-2}$ of emamectin benzoate to monitor insecticide resistance in $C$. includes.

Keywords: Resistance management, monitoring, avermectin, soybean looper.

Chrysodeixis includens (Walker, [1858]) (Lepidoptera: Noctuidae), conhecida como lagarta-falsa-medideira é um dos principais lepidópteros praga em cultivos de soja, algodão, feijão e girassol no Brasil (Carvalho et al. 2012, Guedes et al. 2015). Devido à sua polifagia (Specht et al. 2015), essa espécie pode se desenvolver, simultaneamente, em diferentes plantas hospedeiras.

$O$ controle de $C$. includens é realizado, principalmente, pelo uso de inseticidas químicos e cultivares transgênicas de soja e algodão que expressam proteínas inseticidas de Bacillus thuringiensis (Berliner, 1911). Contudo, no estado de Mato Grosso são cultivados cerca de 60,5 mil hectares de girassol, 56,6 mil hectares de feijão e 220 mil hectares de feijão caupi (Conab 2019), além de pelo menos 3,5 milhões de hectares de cultivares não $B t$ de soja cultivados como área de refúgio estruturado por ser uma estratégia essencial de manejo da resistência de lagartas as proteínas Bt (IRAC-BR 2018). Nessas áreas o controle de $C$. includens é feito principalmente com aplicação de inseticidas químicos.

Casos de resistência de $C$. includens a inseticidas são documentados na literatura aos ingredientes ativos acetato, BHC, DDT, cipermetrina, deltametrina, permetrina, teflutrina, fenvalerato, metomil, paratiom metílico, tiodicarbe e flubendiamida (Owen 2013, IRAC 2019) e baixa suscetibilidade a lambda-cialotrina, teflubenzuron e novaluron (Stacke et al. 2019).

Benzoato de emamectina foi recentemente registrado para o controle de $C$. includens. Este inseticida pertence ao grupo químico das avermectinas enquadrado ao modo de ação 6 (MoA 6) (IRAC 2019). Sua atuação ocorre nos receptores do GABA (ácido gama aminobutírico), induzindo o fluxo de cloro para o interior da célula que resulta na ruptura dos impulsos nervosos paralisando o inseto irreversivelmente e, desse modo, cessando atividade alimentar. Sua atuação se dá por contato e ingestão, sendo a ingestão considerada a principal via de intoxicação (Jansson \& Dybas 1996).

Considerando que o benzoato de emamectina é registrado para $C$. includens nas culturas do feijão e soja, é estratégico o conhecimento dos níveis de suscetibilidade de populações dessa espécie a esse ingrediente ativo. Portanto, objetivou-se nesse estudo estabelecer a linha de suscetibilidade de populações de $\mathrm{C}$. includens a esse inseticida e estimar uma concentração diagnóstica para avaliar a evolução da resistência em futuros monitoramentos.

Para a caracterização da suscetibilidade de $C$. includens a benzoato de emamectina, 10 populações de $C$. includens foram coletadas em importantes regiões produtoras de soja do estado de Mato Grosso na safra agrícola de 2017/2018 (Tab. 1). Em cada local foram coletadas, aproximadamente, 150 lagartas padronizadas por tamanho para a constituição das populações. Além das populações de campo foi avaliada uma população criada em laboratório, em condições controladas, sem exposição a inseticidas, proteínas $B t$ e produtos à base de microrganismos por gerações sucessivas desde 2014.

Tabela 1. Identificação, localização e data de coleta de populações de Chrysodeixis includens em Mato Grosso para caracterizar a suscetibilidade a benzoato de emamectina.

\begin{tabular}{lcccc}
\hline \multicolumn{1}{c}{ População } & $\begin{array}{c}\text { Data da } \\
\text { Coleta }\end{array}$ & $\begin{array}{c}\text { No de } \\
\text { indivíduos } \\
\text { coletados }\end{array}$ & Latitude & Longitude \\
\hline Água Boa & $17 / 01 / 2018$ & 215 & $14^{\circ} 00^{\prime} 21^{\prime \prime}$ & $52^{\circ} 09^{\prime} 01^{\prime \prime}$ \\
\hline Campo Verde & $18 / 01 / 2018$ & 129 & $15^{\circ} 31^{\prime} 30^{\prime \prime}$ & $55^{\circ} 18^{\prime} 02^{\prime \prime}$ \\
\hline Canarana & $16 / 01 / 2018$ & 213 & $14^{\circ} 45^{\prime} 40^{\prime \prime}$ & $52^{\circ} 21^{\prime} 36^{\prime \prime}$ \\
\hline Cláudia & $23 / 01 / 2018$ & 227 & $11^{\circ} 38^{\prime} 40^{\prime \prime}$ & $54^{\circ} 35^{\prime} 50^{\prime \prime}$ \\
\hline Dom Aquino & $18 / 01 / 2018$ & 155 & $15^{\circ} 30^{\prime} 19^{\prime \prime}$ & $54^{\circ} 38^{\prime} 36^{\prime \prime}$ \\
\hline Lucas do Rio Verde & $20 / 01 / 2018$ & 133 & $13^{\circ} 14^{\prime} 95^{\prime \prime}$ & $56^{\circ} 04^{\prime} 87^{\prime \prime}$ \\
\hline Nova Mutum & $12 / 01 / 2018$ & 163 & $13^{\circ} 41^{\prime} 43^{\prime \prime}$ & $55^{\circ} 57^{\prime} 19^{\prime \prime}$ \\
\hline Nova Xavantina & $17 / 01 / 2018$ & 180 & $12^{\circ} 46^{\prime} 34^{\prime \prime}$ & $52^{\circ} 01^{\prime} 14^{\prime \prime}$ \\
\hline Ribeirão Cascalheira & $16 / 01 / 2018$ & 180 & $12^{\circ} 46^{\prime} 34^{\prime \prime}$ & $52^{\circ} 01^{\prime} 14^{\prime \prime}$ \\
\hline União do Sul & $10 / 01 / 2018$ & 128 & $11^{\circ} 28^{\prime} 47^{\prime \prime}$ & $54^{\circ} 04^{\prime} 20^{\prime \prime}$ \\
\hline
\end{tabular}

Para criação das populações, as lagartas foram mantidas em dieta artificial até atingirem a fase de pupa (Parra 2001). Os adultos foram 
mantidos em gaiolas de PVC de 20x25 cm (diâmetro x altura) revestida internamente com papel A4 para oviposição e cobertas por tecido voil. Solução aquosa de sacarose $10 \%$ foi fornecida para alimentação dos adultos. Os papéis contendo os ovos foram substituídos a cada dois dias, e os ovos foram mantidos em sala climatizada (temperatura de $24 \pm 2 \stackrel{\circ}{\circ}$, umidade relativa de $70 \pm 10 \%$ e fotofase de $14 \mathrm{~h}$ ) até a eclosão das lagartas para o início de um novo ciclo de criação ou a realização dos bioensaios.

Os bioensaios foram realizados utilizando o método de contaminação da superfície da dieta artificial com inseticida. Foram utilizadas placas plásticas (Costar ${ }^{\circledR}$, Cambridge, Massachusetts, EUA 24 células com área interna de cada célula igual a 1,91 cm²), contento $1,5 \mathrm{~mL}$ de dieta artificial.

Para estabelecimento das concentrações letais para $50 \%\left(\mathrm{CL}_{50}\right)$ e 99\% $\left(\mathrm{CL}_{99}\right)$ dos indivíduos, realizou-se um bioensaio de curva resposta utilizando a população de laboratório como padrão e 7 concentrações $\left(0,0061,0,0123,0,0245,0,0491,0,0982,0,1963\right.$ e 0,3927 $\mu$ g i.a. $\left.\mathrm{cm}^{2}\right)$ para $\mathrm{CL}_{99}$. O produto utilizado foi Proclaim ${ }^{\circledR} 50$ WG WG; Syngenta Lote YGM3D14005(D).

A fim de estimar uma concentração diagnóstica para futuros monitoramentos, foram analisadas as $\mathrm{CL}_{99}$ para as 11 populações conforme proposto por Bird et al. (2019).

Para o preparo das concentrações, o inseticida foi diluído em água ultrapura com adição de espalhante adesivo Break-thru ${ }^{\circledast}$ na concentração de $0,1 \%(\mathrm{v} / \mathrm{v})$ e, para o tratamento controle utilizou-se apenas água mais espalhante adesivo a 0,1\% (v/v).

Com auxílio de um dispensador automático (Eppendorf Multipette ${ }^{\circledR}$ M4) foi transferido $20 \mu \mathrm{L}$ da solução inseticida com as concentrações testadas para cada célula da placa. As placas foram mantidas em câmaras de fluxo laminar por aproximadamente uma hora até a evaporação do excesso de água. Logo após, uma lagarta em 3 ínstar foi transferida para cada célula com o auxílio de um pincel e as placas foram mantidas em câmara climatizada $(24 \pm 2$ 드, umidade relativa de $70 \pm 10 \%$ e de 14 horas de fotofase) por 24 horas. Após esse período foi avaliada a mortalidade, sendo consideradas mortas às lagartas que não apresentavam movimentos coordenados quando tocadas com um pincel fino.

O delineamento experimental foi inteiramente casualizado com nove repetições de 24 lagartas cada, sendo testadas 216 lagartas por concentração. Os dados de mortalidade obtidos para as populações foram submetidos a análise de Probit através do programa Polo-PC (LeOra Software 1987) o qual apresentou os valores das concentrações letais para 50 e $99 \%\left(\mathrm{CL}_{50}\right.$ e $\left.\mathrm{CL}_{99}\right)$ e seus respectivos intervalos de confiança (I.C. 95\%).

Todas as populações de $C$. includens coletadas em campo apresentaram elevada suscetibilidade ao benzoato de emamectina, inclusive com valores de $\mathrm{CL}_{50}$ inferiores a população de laboratório. Para as dez populações de $C$. includens provenientes do campo as $\mathrm{CL}_{50}$ variaram de 0,015 a $0,065 \mu$ i.a. $\mathrm{cm}^{2}$ e as $\mathrm{CL}_{99}$ variaram de 0,285 a 2,867 $\mu$ g i.a. $\mathrm{cm}^{2}$. A CL50 da população de laboratório foi 0,049 $\mu$ g i.a. $\mathrm{cm}^{2}$ e para CL99 igual a 0,405 $\mu$ g i.a. $\mathrm{cm}^{2}$ (Tab. 2).

Para as populações de campo houve uma variação na $\mathrm{CL}_{50}$ de 0,02 a 0,06 $\mu$ g i.a. $\mathrm{cm}^{-2}$, sendo os valores estimados para Canarana, Cláudia, Nova Mutum e União do Sul de $0,02 \mu \mathrm{g}$ i.a. $\mathrm{cm}^{-2}$ e os maiores para Nova Xavantina e Campo Verde de 0,06 $\mu$ g i.a. $\mathrm{cm}^{-2}$. Para $\mathrm{CL}_{99}$ a variação foi de 0,28 a $2,8 \mu \mathrm{g}$ i.a. $\mathrm{cm}^{-2}$, sendo $0,28 \mu \mathrm{g}$ i.a. $\mathrm{cm}^{-2}$ para o valor de $\mathrm{CL}_{99}$ obtido para Canarana e 2,8 $\mu$ g i.a. $\mathrm{cm}^{-2}$ para Lucas do Rio Verde.

As populações testadas apresentaram coeficientes angulares variando de 1,22 a 2,77 , sendo o menor coeficiente angular $(1,22)$ obtido para a população de Lucas do Rio Verde e o maior valor $(2,77)$ para a população de Nova Xavantina. Entretanto, a razão de resistência entre as populações testadas, foram inferiores a 2 para a $\mathrm{CL}_{50}$ e 7 para a $\mathrm{Cl}_{99}$, indicando que as populações de $C$. includens avaliadas podem ser consideradas como suscetíveis a benzoato de emamectina (Tab. 2).

Stacke et al. (2019) também observaram baixas variações na suscetibilidade de populações de campo de $C$. includens aos inseticidas espinetoram, indoxicarbe, tiodicarbe, clorantraniliprole, flubendiamida e clorfenapir. Entretanto, esses autores constataram baixa suscetibilidade aos inseticidas lambda-cialotrina, metoxifenizide, teflubenzuron e novalurom. $O$ uso preventivo de inseticidas para o controle de pragas pode estar relacionado a essas baixas taxas de suscetibilidade, pois essa estratégia de controle intensifica a pressão de seleção de indivíduos resistentes. Além disso, variações de suscetibilidade entre as populações podem estar associadas a vários fatores como diversidade genética intraespecífica natural, ambientes geográficos distintos, variedades de plantas hospedeiras, métodos de bioensaios, geração testada, tempo de exposição, dietas artificiais, práticas de manejo e fluxo gênico (Zheng et al. 2011, Palma et al. 2015). De acordo com Carriere (2010), mesmo uma pequena variação na suscetibilidade é uma indicação do potencial de seleção da resistência à medida que os insetos se adaptam ao inseticida.

O sistema agrícola de produção em Mato Grosso gera uma exposição repetida de $C$. includens aos inseticidas (incluindo benzoato de emamectina) utilizados no manejo de lepidópteros praga nas culturas de soja, algodão, feijão e girassol que são hospedeiros preferenciais desta desfolhadora (Andrade et al. 2016). Para otimizar o uso desse inseticida no manejo de $C$. includens é necessário estabelecer práticas de rotação com inseticidas de diferentes modos de ação em populações da praga que apresentem baixas razões de resistência, bem como avaliar a adoção de práticas eficientes do manejo integrado de pragas. O uso de plantas Bt (Bernardi et al. 2012), inseticidas a base baculovírus ChinNPV (Muraro et al. 2019) e parasitoides de ovos (Bueno et al. 2009), juntamente ao uso de outros inseticidas químicos podem contribuir para retardar a evolução da resistência.

Considerando que o manejo da resistência é mais efetivo quando implementado de modo preventivo, ou seja, no início da evolução da

Tabela 2. Resposta de concentração-mortalidade $\left(\mathrm{CL} ; \mu \mathrm{g}\right.$ i.a. $\left.\mathrm{cm}^{-2}\right)$ de $C$. includens exposta a benzoato de emamectina.

\begin{tabular}{|c|c|c|c|c|c|c|}
\hline População & $\mathbf{n}$ & $\begin{array}{c}\text { Coef. Angular } \\
\text { ( } \pm \text { EPM) }\end{array}$ & g. $\left.\right|^{c}$ & $\chi^{\mathrm{b}}$ & $\mathrm{CL}_{50}{ }^{\mathrm{a}}\left(\mathrm{I} . \mathrm{C} 95 \% \mu \mathrm{g} \mathrm{cm}^{2}\right)$ & $\mathrm{CL}_{99}{ }^{\mathrm{a}}(\mathrm{I.C} 95 \%)^{\mathrm{a}} \mu \mathrm{g} \mathrm{cm}$ \\
\hline População laboratório & 1080 & $2,540( \pm 0,149)$ & 4 & 4,22 & $0,049(0,042-0,058)$ & $0,405(0,269-0,761)$ \\
\hline Água Boa & 1080 & $1,873( \pm 0,138)$ & 5 & 7,41 & $0,028(0,016-0,038)$ & $0,484(0,257-1,811)$ \\
\hline Campo Verde & 1080 & $2,409( \pm 0,144)$ & 5 & 1,81 & $0,055(0,050-0,061)$ & $0,511(0,400-0,694)$ \\
\hline Canarana & 1080 & $1,834( \pm 0,184)$ & 5 & 3,86 & $0,015(0,007-0,022)$ & $0,285(0,174-0,786)$ \\
\hline Cláudia & 1102 & $1,941( \pm 0,147)$ & 5 & 0,29 & $0,022(0,019-0,025)$ & $0,344(0,254-0,513)$ \\
\hline Dom Aquino & 1520 & $2,140( \pm 0,107)$ & 5 & 2,87 & $0,029(0,027-0,031)$ & $0,354(0,286-0,458)$ \\
\hline Lucas do Rio Verde & 1080 & $1,218( \pm 0,102)$ & 3 & 4,89 & $0,035(0,023-0,049)$ & $2,867(0,982-24,169)$ \\
\hline Nova Mutum & 1080 & $1,449( \pm 0,124)$ & 5 & 11,2 & $0,015(0,004-0,026)$ & $0,618(0,233-12,387)$ \\
\hline Nova Xavantina & 1080 & $2,772( \pm 0,204)$ & 5 & 7,9 & $0,065(0,048-0,083)$ & $0,448(0,262-1,402)$ \\
\hline Ribeirão Cascalheira & 1080 & $2,241( \pm 0,127)$ & 5 & 16,02 & $0,047(0,029-0,069)$ & $0,395(0,314-0,537)$ \\
\hline União do Sul & 1081 & $1,800( \pm 0,121)$ & 5 & 2,48 & $0,016(0,013-0,018)$ & $0,309(0,232-0,446)$ \\
\hline
\end{tabular}

${ }^{a} \mathrm{CL}_{5}$ : concentração de benzoato de emamectina necessária para matar $50 \%$ dos indivíduos durante o período observado de $24 \mathrm{~h}$. Similarmente, $\mathrm{CL}_{99}$ é a concentração necessária para matar $99 \%$ dos indivíduos. ${ }^{\mathrm{b}}$ Teste de qui-quadrado. $\mathrm{P}<0,05 .{ }^{\mathrm{C}} \mathrm{Graus}$ de liberdade 
resistência (Roush \& Mckenzie 1987) e o fato das populações de $C$. includens avaliadas neste trabalho apresentarem alta suscetibilidade ao benzoato de emamectina é essencial o monitoramento contínuo da suscetibilidade, para poder identificar a evolução da resistência ao longo dos anos, de preferência de forma rápida, precisa, prática e compatível para uso em grande escala como o método de concentração diagnóstica (Onstad 2014).

Baseados em nossos resultados, sugerimos que seja utilizado a concentração de $2,867 \mu \mathrm{g}$ i.a. $\mathrm{cm}^{2}$ de benzoato de emamectina para discriminar entre $C$. includens resistentes e suscetíveis.

\section{Contribuição dos Autores}

NRN: conduziu os ensaios e escreveu o manuscrito; FTRF conduziu os ensaios, definiu o método experimental e escreveu o manuscrito; LVT conduziu os ensaios e analisou os resultados; JDNC escreveu o manuscrito; RMP elaborou a hipótese do trabalho, coletou as populações, definiu o método experimental e escreveu o manuscrito.

\section{Referências}

Andrade, K.; Bueno, A. D. F.; da Silva, D. M.; Stecca, C. D. S.; Pasini, A.; Oliveira, M. C. N. de. (2016). Bioecological characteristics of Chrysodeixis includens (Lepidoptera: Noctuidae) fed on different hosts. Australian Entomology, 55(4): 449-454. doi 10.1111/ aen.12208

Bernardi, O.; Malvestiti, G. S.; Dourado, P. M.; Oliveira, W. S.; Martinelli, S.; Berger, G. U.; Head, G. P.; Omoto, C. (2012). Assessment of the high-dose concept and level of control provided by MON 8777701 x MON 89788 soybean against Anticarsia gemmatalis and Pseudoplusia includens (Lepidoptera: Noctuidae) in Brazil. Pest Managment Sciense, 68(7): 1083-1091. doi: 10.1002/ps.3271

Bird, L. J.; Walker, P. W. (2019). Baseline Susceptibility of Helicoverpa punctigera (Lepidoptera: Noctuidae) to indoxacarb, emamectin benzoate, and chlorantraniliprole. Journal of Economic Entomology, 112(2): 818-826. doi: 10.1093/jee/toy389

Bueno, R. C. O. F.; Parra, J. R. P.; Bueno, A. F.; Haddad, M. (2009). Performance of trichogrammarids as biocontrol agentes of Pseudoplusia includens Walker (Lepidoptera: Noctuidae). Neotropical Entomology, 38(3): 389-394. doi: 10.1590/S1519566X2009000300015

Carriere, Y.; Crowder, D. W.; Tabashnik, B. E. (2010). Evolutionary ecology of insect adaptation to Bt crops. Evolutionary Applications, 3(5-6): 561-573. doi: 10.1111/j.1752-4571.2010.00129.x

Carvalho, L. C.; Ferreira, F. M.; Bueno, N. M. (2012). Importância econômica e generalidades para o controle da lagarta-falsamedideira na cultura da soja. Enciclopédia Biosfera, 8(15): 10211034.

Conab - Campanha nacional do Abastecimento. (2018). Acompanhamento da safra brasileira de grãos, Safra 2018/19 Segundo levantamento. <https://www.conab.gov.br/info-agro/ safras/graos/boletim-da-safra-de-graos>. Access em: ix.2018.

Guedes, J. V. C.; Perini, C. R.; Stacke, R. F.; Curioletti, L. E.; Arnemann, J. A.; Alende, V. P. (2015). Lagartas da soja: das lições do passado ao manejo do futuro. Revista Plantio Direto, 24(144): 10-22.

IRAC - Arthropod Pesticide Resistance Database. (2019). <https://www. pesticideresistance.org/display. php?page=species\&arld=248>. Access on: xi.2019.

IRAC-BR, Comitê Brasileiro de Ação à Resistência de Inseticidas. (2018). Manejo da Resistência a Inseticidas e Plantas Bt. <https://www. irac-br.org>. Access on: viii.2019.

Jansson, J. A.; Dybas, R. A. (1996). Avermectins, biochemical mode of action, biological activity, and agricultural importance. In: Ishaaya, I. (Ed.), Insecticides with Novel Modes of Action: Mechanism and Application, pp. 152-170. Springer: New York.

LeOra Software (2002). Polo-plus Probit and logit analysis computer version 1.0. LeOra Software, Berkeley, CA.

Muraro, D. S.; Giacomlli, T.; Stacke, R. F.; Godoy, D. N.; Marçon, P. Popham, H. J. R.; Bernardi, O. (2019). Baseline susceptibility of
Brazilian populations of Chrysodeixis includens (Lepidoptera: Noctuidae) to $C$. includens nucleopolyhedrovirus (ChinNPV) and diagnostic concentration for resistance monitoring. Journal of Economic Entomology, 112(1): 349-354. doi: 10.1093/jee/toy361

Onstand, D. W.; Gassman, A. J. (2014). Concepts and complexities of population genetics. In: Onstand, D. W. (Ed.), Insect Resistance Management, pp 149-177. Elsevier: Amsterdan.

Owen, N. L.; Catchot, A. L.; Musser, F. R.; Gore, J.; Cook, D. C; Jackson, R. (2013). Susceptibility of Chrysodeixis includens (Lepidoptera: Noctuidae) to Reduced-Risk Insecticides. Florida Entomologist, 96(2): 554-559. doi: 10.1653/024.096.0221

Palma, J.; Maebe, K.; Guedes, J. V. C.; Smagghe, G. (2015). Molecular variability and genetic structure of Chrysodeixis includens (Lepidoptera: Noctuidae), an important soybean defoliator in Brazil. PloS One, 10: 1-13. doi: 10.1371/journal.pone.0121260

Parra, J. R. P. (2001). Técnicas de criação de insetos para programas de controle biológico. Piracicaba: ESALQ/FEALQ.

Roush, R. T.; Mckenzie, J. A. (1987). Ecological genetics of insecticide and acaricide resistance. Annual Review of Entomology, Stanford, 32(1): 361-380. doi: 10.1146/annurev.en.32.010187.002045

Specht, A.; Paula-Moraes, S. V. DE; Sosa-Gómez, D. R. (2015). Host plants of Chrysodeixis includens (Walker) (Lepidoptera, Noctuidae, Plusiinae). Revista Brasileira de Entomologia, 59(4): 343-345. doi: 10.1016/j.rbe.2015.09.002

Stacker, R. F.; Giacomelli, T.; Bronzatto, E. S.; Halberstadt, S. A.; Garlet, C. G.; Muraro, D. S.; Guedes, J. V. C.; Bernardi, O. (2019). Susceptibility of Brazilian Populations of Chrysodeixis includens (Lepidoptera: Noctuidae) to Selected Insecticides. Journal of Economic Entomology, 112(3): 1378-1387. doi: 10.1093/jee/toz031 Zheng, X. S.; Ren, X. B.; Su, J. Y. (2011). Insecticide Susceptibility of Cnaphalocrocis medinalis (Lepidoptera: Pyralidae) in China. Journal of Economic Entomology, 104(2): 653-658. doi: 10.1603/EC10419 